\title{
Pepsin in bronchoalveolar lavage fluid: a specific and sensitive method of diagnosing gastro-oesophageal reflux-related pulmonary aspiration.
}

Farrell, S., McMaster, C., Gibson, D., Shields, M. D., \& McCallion, W. A. (2006). Pepsin in bronchoalveolar lavage fluid: a specific and sensitive method of diagnosing gastro-oesophageal reflux-related pulmonary aspiration. Journal of Pediatric Surgery, 41(2), 289-293. https://doi.org/10.1016/j.jpedsurg.2005.11.002

Link to publication record in Ulster University Research Portal

Published in:

Journal of Pediatric Surgery

Publication Status:

Published (in print/issue): 01/01/2006

DOI:

10.1016/j.jpedsurg.2005.11.002

Document Version

Publisher's PDF, also known as Version of record

\section{General rights}

Copyright for the publications made accessible via Ulster University's Research Portal is retained by the author(s) and / or other copyright owners and it is a condition of accessing these publications that users recognise and abide by the legal requirements associated with these rights.

\section{Take down policy}

The Research Portal is Ulster University's institutional repository that provides access to Ulster's research outputs. Every effort has been made to ensure that content in the Research Portal does not infringe any person's rights, or applicable UK laws. If you discover content in the Research Portal that you believe breaches copyright or violates any law, please contact pure-support@ulster.ac.uk. 


\title{
BAPS Prize
}

\section{Pepsin in bronchoalveolar lavage fluid: a specific and sensitive method of diagnosing gastro-oesophageal reflux-related pulmonary aspiration}

\author{
Stephen Farrell ${ }^{a}{ }^{*}$, Cyril McMaster $^{a}$, David Gibson ${ }^{b}$, Michael D. Shields $^{a}$, \\ William A. McCallionc
}

\author{
${ }^{a}$ Department of Child Health, Queen's University Belfast, Royal Victoria Hospital, Institute of Clinical Science, \\ Belfast, BT12 6BJ Northern Ireland, UK \\ ${ }^{\mathrm{b}}$ Department of Vision Sciences, Queen's University Belfast, Northern Ireland, Belfast, BT12 6BE Northern Ireland, UK \\ ${ }^{\mathrm{c}}$ Department of Paediatric Surgery, Royal Belfast Hospital for Sick Children, BT12 6 BE Northern Ireland, UK
}

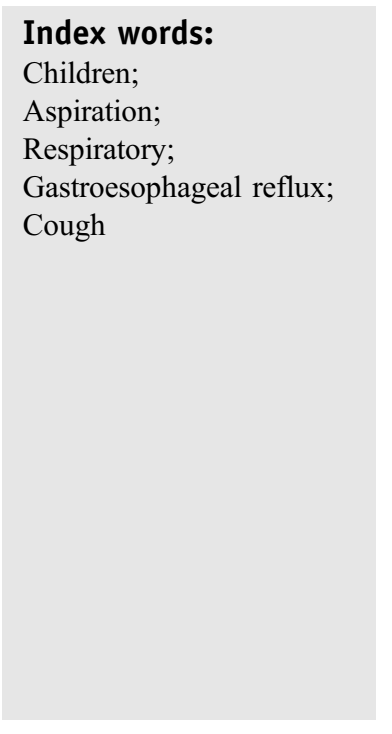

\begin{abstract}
Objectives: Gastro-oesophageal reflux (GOR)-related aspiration is associated with respiratory disease, but the current "gold standard" investigation, the lipid-laden macrophage index (LLMI), is flawed. A specific marker of GOR-related aspiration should originate in the stomach, but not the lung. An assay to detect gastric pepsin in the bronchoalveolar lavage (BAL) of children was developed and validated. Methods: Gastro-oesophageal reflux was diagnosed in 33 children using intra-oesophageal $\mathrm{pH}$ monitoring. Thirteen asymptomatic negative controls requiring endotracheal intubation for elective surgery and 5 positive control patients with observed aspiration were recruited. All subjects received a BAL; the fluid obtained was analysed for the pepsin content and the LLMI.

Results: All subjects in the negative control group were negative for pepsin. The positive control group had a significantly greater median pepsin level $(P<.01)$ compared with negative controls. Patients with proximal oesophageal GOR and chronic cough also had significantly elevated pepsin levels $(P=.04)$. The LLMI was not significantly elevated by the presence of cough or GOR.

Conclusions: This study suggests that GOR-related aspiration plays a role in chronic cough in children with known GOR. Detecting pepsin in BAL fluid may therefore become an important adjunct in patient selection for antireflux surgery.

(C) 2006 Elsevier Inc. All rights reserved.
\end{abstract}

Gastro-oesophageal reflux (GOR), which is physiologic in most children, becomes pathologic if associated with complications such as failure to thrive, oesophagitis, or

Presented at the 52nd Annual Congress of British Association of Paediatric Surgeons, Dublin, Ireland, July 12-15, 2005.

* Corresponding author. Tel.: +44 2890632568; fax: +44 2890236455.

E-mail address: s.farrell@qub.ac.uk (S. Farrell). respiratory disease. Although antireflux surgery is required for the minority, it is still the third most common major operation carried out in children [1]. The most common indication for antireflux surgery is reflux-related respiratory disease [2]. However, proving cause and effect in many cases is problematic.

The association between GOR and respiratory disease was first described by Sir William Osler, in 1892 [3]. 
However, it is only over the past 2 decades that evidence of a causal relationship has emerged. In 1986, Buts et al [4] used 24-hour intra-oesophageal $\mathrm{pH}$ monitoring to diagnose GOR in $61 \%$ of children with varying respiratory conditions, whereas Andze et al, in 1991, published a large landmark study of 500 children investigated primarily for respiratory complaints and found significant GOR in 63\% [5]. Furthermore, improvement in respiratory symptoms after antireflux surgery has been documented.

The relationship between GOR and respiratory disease is complex, and 2 theories by which GOR may cause respiratory symptoms have been proposed:

1. a vagus nerve-mediated reflex arc, the "reflex" theory;

2. aspiration of gastric contents into the tracheobronchial tree, the "reflux" theory.

The reflex theory involves acid exposure to the lower oesophagus that stimulates the vagus nerve resulting in laryngospasm, bronchospasm, or central apnoea via a reflex arc, which is thought to arise because of the common embryological origins of the oesophagus and tracheobronchial tree. Evidence for this theory was provided by Mansfield et al [6] in an animal model whereby increased airway resistance in response to the intra-oesophageal acid was abolished by bilateral vagotomy. Evidence supporting this vagal-mediated reflex is, however, limited in the paediatric population.

The reflux theory describes a process whereby gastric material is aspirated into the tracheobronchial tree producing an airway response. Although macroaspiration is usually clinically obvious, recurrent microaspiration is notoriously difficult to diagnose.

The most widely used method for diagnosing GORrelated pulmonary aspiration is the detection of lipid in alveolar macrophages. It is proposed that extrinsic food lipid is refluxed, aspirated into the lungs, and phagocytosed by alveolar macrophages. The engulfed lipid can then be demonstrated by staining cells obtained by bronchoalveolar lavage (BAL) with oil red O. A potential problem is the breakdown of intrinsic alveolar phospholipids in patients with chronic lung disease resulting in a false-positive result. To improve specificity, Corwin and Irwin [7] described a lipid-laden macrophage index (LLMI), quantifying the numbers of macrophages laden with lipid. They reported a sensitivity of $100 \%$ for pulmonary aspiration, but the specificity was only $57 \%$. Furthermore, several studies have questioned the diagnostic accuracy of the LLMI in diagnosing recurrent GOR-related microaspiration. Both Kazachkov et al [8] and Krishnan et al [9] found that the mean LLMI of children with GOR and respiratory disease was not significantly greater than those with neither, whereas Knauer-Fischer and Ratjen [10] demonstrated that children with respiratory disease but no GOR had significantly elevated LLMI scores compared with healthy controls.
As the current "gold standard" for diagnosing pulmonary aspiration, the LLMI is clearly flawed. The aim of this project was to establish a specific and sensitive marker of GOR-related pulmonary aspiration. Such a marker should fulfil a number of criteria.

1. It should not normally be found in the tracheobronchial tree or lungs.

2. It should only be present within gastric secretions.

3. It should remain biologically active or detectable after aspiration.

4. It should be physically retrievable from the lungs using minimally invasive procedures.

Gastric pepsin is one such compound that appears to fulfil these criteria. It is a proteolytic enzyme secreted by the gastric chief cells and mucus neck cells as inactive pepsinogen. This is cleaved at $\mathrm{pH} 5$ to form the active pepsin. Because pepsinogen is secreted by cells unique to gastric mucosa and requires acidic conditions for the final step of production, the detection of pepsin in bronchoalveolar fluid would theoretically be highly specific for GORrelated aspiration.

We sought to develop and validate an immunoassay to detect pepsin in BAL specimens obtained from children to differentiate those patients with respiratory symptoms secondary to GOR-related pulmonary aspiration from those with a chronic respiratory disorder, such as atopic asthma.

\section{Methods}

Three groups of children younger than 14 years were recruited.

1. Study group - children undergoing general anaesthesia as part of their routine clinical investigations for symptoms of GOR, with or without respiratory symptoms.

2. Positive control group - children intubated in the paediatric intensive care unit who had a proven macroscopic aspiration event, that is, with milk having been suctioned from the endotracheal tube after nasogastic feeding.

3. Negative control group - children, with no history of GOR or respiratory disease, undergoing general anaesthesia with endotracheal intubation for elective surgery.

Each patient underwent a BAL using a sterile suction catheter passed down the endotracheal tube and wedged in a bronchus with gentle pressure. The lavage was performed by instilling 1 to $2 \mathrm{~mL} / \mathrm{kg}$ of sterile normal saline via the catheter and aspirating as much fluid as possible. The lavage fluid obtained was centrifuged producing a cellular pellet and supernatant fluid. The cellular pellet was used to 
produce a slide for oil red O staining and LLMI assessment, and the supernatant fluid was analysed with a pepsin assay.

In addition, patients in the study group underwent upper gastrointestinal endoscopy followed by 24-hour dual-channel $\mathrm{pH}$ monitoring using the Digitrapper $\mathrm{pH} 400$ system (Medtronic Functional Diagnostics A/S, Skovlunde, Denmark). Diagnosis of distal GOR was made in infants younger than 1 year if the $\mathrm{pH}$ at the distal oesophageal probe was less than 4 for greater than $12 \%$ of the study time. For children older than 1 year, a cutoff value of $6 \%$ was used. The diagnosis of proximal GOR was made in all subjects if the $\mathrm{pH}$ at the proximal oesophageal probe was less than 4 for greater than $1 \%$ of the study time.

A pepsin assay was developed based on a sandwich enzyme-linked immunosorbent assay (ELISA) principle. Using 2 primary antiporcine pepsin antibodies, the first bound to the surface of the ELISA microplate wells, captured the target antigen (present within the BAL supernatant) binding it to the well surface. After wash steps the amount of captured antigen was quantified by the addition of a second antiporcine pepsin antibody, labelled with the enzyme horseradish peroxidase. A substrate, tetramethylbenzidine, when added was acted upon by any bound horseradish peroxidase producing a colour change, which was measured and compared with a reference standard curve.

A number of defined validation parameters were used to evaluate this developed assay. The mean intra-assay coefficient of variation (CV) was $5.9 \%$ with a mean interassay $\mathrm{CV}$ of $6.4 \%$. The mean percentage of recovery rate (target value is $100 \%$ ) for pepsin in BAL fluid was $88.2 \%$, and the mean assay linearity was $105.9 \%$. Western blot was used to establish cross-reactivity of the antiporcine pepsin antibodies with human gastric fluid. No assay crossreactivity was detected with any of the serum proteins tested; it did however display cross-reactivity with pepsinogen, but not with another aspartic protease chymosin or rennin. None of the pepsin-positive samples had evidence of pepsinogen 1 when tested, using a commercially available ELISA (Biohit Ltd, Torquay, UK).

The cellular pellet obtained from the BAL fluid was used to prepare a cytospin slide, which was incubated in filtered oil red O. Excess oil red O stain was removed, the slides gently washed with distilled water and counterstained with Harris haematoxylin before being mounted. The slides were then scored microscopically; 100 consecutive alveolar macrophages were assessed for the amount of intracytoplasmic lipid and assigned a score of 1 to 4 , producing a potential maximum of 400 .

Descriptive statistics were used to calculate the median, interquartile range (IQR), and range. Correlations between factors were tested using the 2-tailed Spearman correlation coefficient. The difference in proportions of subjects testing positive for pepsin was tested by Pearson $\chi^{2}$ test. The differences in levels of pepsin and LLMI scores detected between 2 groups were tested by the 2-tailed Mann-Whitney $U$ test. Differences in levels of pepsin and the LLMI scores detected between subgroups and the control group were tested using the Kruskal-Wallis multiple comparison $z$ value test; $z$ value greater than 1.96 was regarded as significant for an individual 2-group comparison. Statistical significance was accepted as a $P$ value of less than .05 .

\section{Results}

Samples from 74 patients (median age, 5.3 years; IQR, 2.1-9.1 years) were assayed and included in the analysis. The overall median pepsin level was $0 \mathrm{ng} / \mathrm{mL}$ (IQR, $0-1.5 \mathrm{ng} / \mathrm{mL}$; range, $0-100 \mathrm{ng} / \mathrm{mL}$ ), and median LLMI score was 14.5 (IQR, 3.7-44.1; range, 0-137). Fifty-six subjects were recruited into the study group, 5 into the positive control group, and 13 into the negative control group. There was no significant difference in proportion of subjects with pepsin detected $(P=.82)$, the median pepsin levels $(P=.78)$, or the median LLMI score $(P=.88)$ between males $(\mathrm{n}=53)$ and females, and there was no significant correlation of pepsin levels or LLMI scores with age $(r=-0.15, P=.09$ and $r=$ $0.05, P=.65)$. There was no significant correlation between the BAL pepsin level and the LLMI score $(r=0.13, P=.30)$.

There was a significantly greater proportion of subjects in the positive control (pulmonary aspiration) group with pepsin detected in their BAL than in the negative control group $(P<.01)$. Likewise, the median BAL pepsin level was significantly greater in the positive control group than in the negative control group (median, 0 vs $24.13 \mathrm{ng} / \mathrm{mL}$; $P<.01$ ). The sensitivity, specificity, and positive and negative predictive values for the pepsin assay to detect pulmonary aspiration were $80 \%, 100 \%, 100 \%$, and $93 \%$, respectively. The median LLMI score was also significantly elevated in the positive control group (44.0 vs $16.75, P<.01)$.

Children were assigned to groups depending on the presence of distal oesophageal GOR only (group A, $n=9$ ), proximal oesophageal GOR only (group $\mathrm{B}, \mathrm{n}=15$ ), and distal combined with proximal GOR (group $\mathrm{C}, \mathrm{n}=9$ ). Group B was the only group with a significantly elevated pepsin level compared with the control population $(z=2.33$, indicating a significant difference in group medians; $P<$ $.05)$. It was also associated with an increased proportion of subjects positive for pepsin compared with the negative controls $(P=.02)$. When groups $\mathrm{B}$ and $\mathrm{C}$ were combined into a single group of subjects with proximal GOR \pm distal GOR, and the analysis repeated, the combined group was no longer significantly associated with elevated pepsin levels in the BAL $(z=1.88, P>.05)$. There was, however, still a significantly greater proportion of patients positive for pepsin in the combined group compared with the negative controls $(P=.04)$. The LLMI was not significantly elevated in any of the GOR groups. Patients within the study group were further classified by the presence of respiratory symptoms. Those subjects with proximal GOR (groups B and $\mathrm{C}$ combined) who also complained of chronic cough had significantly greater levels of pepsin detected compared 
with the negative control group $(P=.04)$, whereas the pepsin levels in those with proximal GOR and no cough were not significantly different from the negative controls $(P=.08)$. Similarly, the presence of cough in patients negative for GOR on $\mathrm{pH}$ monitoring was significantly associated with increased BAL levels of pepsin, compared with the controls $(P<.01)$. The LLMI was not significantly elevated in any of these groups.

\section{Discussion}

Gastro-oesophageal reflux-associated respiratory disease is a myriad, and proving cause and effect is problematic. Antireflux surgery is now the third most common major operation carried out in the paediatric population, and respiratory disease is the most common indication. A significant problem, however, for paediatric surgeons is patient selection as diagnosing pulmonary aspiration has been notoriously difficult.

Respiratory symptoms associated with GOR represent a "chicken and egg" dilemma. Does the reflux cause respiratory disease or vice versa? In addition to the 2 mechanisms whereby reflux causes respiratory symptoms (the reflex and reflux theories), there is evidence that respiratory disease can induce secondary GOR. For example, methacholine-induced airway obstruction is associated with increased GOR [11]. It is therefore clear that a complex relationship between respiratory disease and GOR exists. This makes it difficult to clinically differentiate those patients who are aspirating refluxed gastric contents and would benefit from antireflux surgery from those with GOR induced by preexisting respiratory disease. This clinical dilemma is exacerbated by the flaws, highlighted in this and other studies, in the currently accepted gold standard-the LLMI. Although the median LLMI was significantly elevated in the positive control group when compared with the negative controls, only one of the subjects with proven aspiration had a LLMI greater than the previously suggested cutoff value of 100 for pulmonary aspiration [12]. Furthermore, the LLMI did not differentiate refluxing patients with or without respiratory disease from normal controls, in keeping with the findings of both Kazachkov et al [8] and Krishnan et al [9].

Assays to detect pepsin are based on 2 separate principles, the proteolytic activity and the antigenic property of pepsin. Proteolytic assays depend on the estimation of the number of peptide bonds broken during the reaction between pepsin and a protein substrate, whereas an immunoassay utilises the antigenic properties of a protein, allowing its identification through an antigen-antibody reaction. Given that the proteolytic activity of pepsin is greatest at $\mathrm{pH} 2$, but decreases with increasing $\mathrm{pH}$, there is likely to be a reduction in the proteolytic activity of pepsin in $\mathrm{pH}$-neutral BAL. The use of a pepsin proteolytic assay to investigate BAL may therefore be problematic. This has been highlighted in 2 studies. In an animal model, the proteolytic activity of pepsin in BAL obtained from rabbits, after induced gastric fluid aspiration, decreased by $37 \%$ within 1 hour of aspiration [13]. Furthermore, in a study of 65 children, middle ear effusions were tested for pepsin, using both a proteolytic assay and an immunoassay, to investigate the role of GOR in the causation of otitis media. The immunoassay was positive in $91 \%$ of effusions tested compared with only $31 \%$ positive with the proteolytic assay. It is therefore reasonable to assume that the more alkaline conditions present in the lung may reduce or completely abolish the proteolytic activity of aspirated pepsin, lowering the assay sensitivity [14].

It was necessary to develop an "in-house" immunoassay for this study because of the lack of a commercially available antihuman pepsin antibody. To overcome this, the antiporcine pepsin antibodies used herein were demonstrated to detect a protein in human gastric fluid with the same molecular weight as pepsin. This in-house assay detected pepsin in the BAL fluid of 4 of 5 subjects in the positive control group (sensitivity, $80 \%$ ), whereas none of the 13 negative control subjects had pepsin detected (specificity, 100\%). This was compared with the LLMI, performed on the same subjects, which gave an unacceptably low sensitivity of $20 \%$ but a specificity of $100 \%$.

This is the first study to investigate reflux-related pulmonary aspiration using a pepsin immunoassay, with comparison to the currently accepted gold standard (LLMI) and with the recruitment of both positive and negative control groups. When the pepsin concentration in the BAL fluid of children with proximal oesophageal GOR was analysed, those patients with chronic cough had significantly greater levels than negative controls. There was, however, no such difference when children with proximal GOR but no cough were compared with negative controls. This suggests that reflux-related aspiration plays a significant role in the pathogenesis of respiratory symptoms in patients with proximal GOR. The fact that not all patients with proximal GOR had pepsin detected in the BAL suggests that the upper oesophageal sphincter or laryngeal protective reflexes prevented aspiration in some of this cohort. The finding that chronic cough is also associated with elevated BAL pepsin levels in children who present with GOR symptoms but whose $\mathrm{pH}$ data are within normal limits would also support the reflux theory for related respiratory disease. This perhaps highlights the deficiencies of $\mathrm{pH}$ monitoring used to investigate reflux-related pulmonary aspiration, as reflux events to the larynx may occur and result in aspiration and respiratory disease, but may not occur frequently enough or be sufficiently acidic for the patients to be diagnosed with GOR.

Correct patient selection for antireflux surgery is crucial. However, until now, proving aspiration in children with GOR and respiratory disease was difficult. The currently accepted gold standard investigation (LLMI) is flawed. In this study, a pepsin ELISA was developed and found to be specific and sensitive for diagnosing pulmo- 
nary aspiration. Detecting pepsin in BAL fluid should therefore become an important adjunct in patient selection for antireflux surgery.

\section{References}

[1] Fonkalsrud EW, Ament ME. Gastroesophageal reflux in childhood. Curr Probl Surg 1996;33:1-70.

[2] Fonkalsrud EW, Bustorff-Silva J, Perez CA, et al. Antireflux surgery in children under 3 months of age. J Pediatr Surg 1999;34:527-31.

[3] Sontag SJ. Gastroesophageal reflux disease and asthma. J Clin Gastroenterol 2000;30:S9-S30.

[4] Buts JP, Barudi C, Moulin D, et al. Prevalence and treatment of silent gastro-oesophageal reflux in children with recurrent respiratory disorders. Eur J Pediatr 1986;145:396-400.

[5] Andze GO, Brandt ML, St Vil D, et al. Diagnosis and treatment of gastroesophageal reflux in 500 children with respiratory symptoms: the value of $\mathrm{pH}$ monitoring. J Pediatr Surg 1991;26:295-9.

[6] Mansfield LE, Hameister HH, Spaulding HS, et al. The role of the vague nerve in airway narrowing caused by intraesophageal hydrochloric acid provocation and esophageal distention. Ann Allergy 1981;47:431 - 4 .
[7] Corwin RW, Irwin RS. The lipid-laden alveolar macrophage as a marker of aspiration in parenchymal lung disease. Am Rev Respir Dis 1985;132:576-81

[8] Kazachkov MY, Muhlebach MS, Livasy CA, et al. Lipid-laden macrophage index and inflammation in bronchoalveolar lavage fluids in children. Eur Respir J 2001;18:790 - 5 .

[9] Krishnan U, Mitchell JD, Tobias V, et al. Fat laden macrophages in tracheal aspirates as a marker of reflux aspiration: a negative report. J Pediatr Gastroenterol Nutr 2002;35:309-13.

[10] Knauer-Fischer S, Ratjen F. Lipid-laden macrophages in bronchoalveolar lavage fluid as a marker for pulmonary aspiration. Pediatr Pulmonol 1999;27:419-22.

[11] Moote DW, Lloyd DA, McCourtie DR, et al. Increase in gastroesophageal reflux during methacholine-induced bronchospasm. J Allergy Clin Immunol 1986;78:619-23.

[12] Adams R, Ruffin R, Campbell D. The value of the lipid-laden macrophage index in the assessment of aspiration pneumonia. Aust NZ J Med 1997;27:550-3

[13] Badellino MM, Buckman Jr RF, Malaspina PJ, et al. Detection of pulmonary aspiration of gastric contents in an animal model by assay of peptic activity in bronchoalveolar fluid. Crit Care Med 1996; 24:1881-5.

[14] Tasker A, Dettmar PW, Panetti M, et al. Is gastric reflux a cause of otitis media with effusion in children? Laryngoscope 2002;112:1930 - 4 . 